\title{
Respostas Diferenciais ao Playback em Levantamento de Callithrix aurita na Microrregião de Viçosa/MG
}

Natan Tomaz Massardi ${ }^{1}$, Orlando Vítor Vital, Samuel Lucas Brasileiro Silvério, Fernanda de Fátima Rodrigues da Silva ${ }^{1}$, Fabiano Rodrigues de $\mathrm{Melo}^{1}$, \& Leandro Jerusalinsky ${ }^{2}$

${ }^{1}$ Universidade Federal de Viçosa/UFV, Brasil. < natan.massardi@ufv.br, fernanda.fatima@ufv.br, frmelo@ufv.br>
${ }^{2}$ Instituto Chico Mendes de Conservação da Biodiversidade/CPB/ICMBio, Brasil. <jerusalinsky@icmbio.gov.br>

RESUMO - Callithrix aurita é um pequeno primata endêmico da Mata Atlântica brasileira e considerado "em perigo" de extinção (EN) devido à perda e fragmentação de seus habitat e à competição e hibridação com congêneres invasores. Nesse contexto, identificar fragmentos florestais habitados por Callithrix spp. é uma das estratégias prioritárias para a sua conservação. Como as populações de $C$. aurita podem estar subamostradas pelas diversas técnicas de detecção, o objetivo deste trabalho foi avaliar a eficiência do playback como método auxiliar à busca ativa de Callithrix spp. em fragmentos da microrregião de Viçosa. Para tal, considerou-se a taxa de resposta de Callithrix spp. em relação ao tamanho do fragmento e o horário do dia. Quinze fragmentos foram vistoriados, com 88 pontos de playback. Não houve registro de $C$. aurita, mas houve resposta de Callithrix spp. em 5,68\% das execuções. As respostas foram obtidas entre 07h - 08h ou entre $16-17 \mathrm{~h}$. As maiores taxas de resposta de Callithrix spp. ocorreram em fragmentos maiores que 100ha, seguido por fragmentos entre 50 ha e 100 ha, e por fragmentos menores que 50 ha. O horário do dia está intimamente ligado à temperatura; portanto, os horários de maior responsividade ao playback são corroborados pelo fato de haver perdas abaixo do esperado na propagação do som. Não houve interferência significativa da área do fragmento e do horário nas respostas de Callithrix spp. Assim, a técnica de playback demonstrou-se útil para levantamentos de Callithrix, independentemente de seu tamanho, em especial quando executado no início do dia ou fim da tarde.

Palavras-chave: Sagui-da-serra-escuro; fragmentação; vocalização; survey; espécies invasoras.

\section{Differential Responses to Playback in a Callithrix aurita Survey in the Microregion of Viçosa/MG}

ABSTRACT - Callithrix aurita is an endemic primate from brazilian Atlantic Rainforest. The species is classified as "endangered" (EN) by the Brazilian Red Book of Endangered Fauna and the IUCN Red List of Threatened Species ${ }^{\mathrm{TM}}$. Thus, the mapping and identification of forest fragments inhabited by $C$. aurita populations, their invasive and hybrid counterparts are one of the priority strategies for its conservation. As populations of $C$. aurita may be subsampled by traditional census techniques, the evaluation of these techniques is essential. The present work evaluated the efficiency of playback in considering the response rate from Callithrix spp. with the fragment size and hour of day, using active search with playback assistance in fragments in the micro-region of Viçosa. Fifteen fragments were inspected, with 88 playback points. There were no records of $C$. aurita, but there was a response from Callithrix spp. in 5.68\% of executions. All responses were obtained between 7:00 am and 8:00 am or between 4:00 pm and 5:00 pm. The highest meeting rates of Callithrix spp. occurred in fragments larger than $100 \mathrm{ha}$, followed by fragments between $50 \mathrm{ha}$ and $100 \mathrm{ha}$, followed by fragments smaller than 50ha. The hour of day is closely linked to the temperature, so the prevalence of responses in the early hours of the morning and at dusk in forest environments is corroborated by the fact that there are losses below the expected in sound propagation at those periods. There was no significant interference from the fragment area and time in the responses of Callithrix spp.

Keywords: Buffy-tufted-ear marmoset; fragmentation; vocalization; survey; invasive species. 


\title{
Respuestas Diferenciales a la Reproducción de Playback en un Relevamiento Poblacional de Callithrix sp. en la Microrregión de Viçosa/MG
}

\begin{abstract}
RESUMEN - El tití Callithrix aurita es un primate endémico del Bosque Atlántico y está en peligro de extinción. Así, el mapeo e identificación de fragmentos de bosque con presencia de esta especie, asimismo como de invasores o híbridos es una estrategia prioritaria para su conservación. Como las poblaciones de $C$. aurita pueden estar submuestreadas por las técnicas de detección tradicionales, es esencial evaluar la efectividad de la técnica empleada. El presente trabajo tuvo como objetivo evaluar la efectividad de la reproducción de playback con relación a la tasa de respuesta de Callithrix spp., considerando el tamaño del fragmento y el tiempo, como método auxiliar a la búsqueda activa en fragmentos en la microrregión de Viçosa, MG. Se examinaron quince fragmentos, con 88 puntos de reproducción. Hubo una respuesta de Callithrix spp. en el 5,68\% de las reproducciones. Todas las respuestas se obtuvieron entre 07:00-08:00h y 16:00-17:00h. Las tasas de respuesta más altas para Callithrix spp. ocurrieron en fragmentos mayores que 100 acres, seguidos de fragmentos entre 50 y 100 acres y fragmentos menores de 50 hectáreas. La hora del día está fuertemente relacionada con la temperatura; la prevalencia de respuestas en las primeras horas de la mañana y en el atardecer, en ambientes forestales, se corrobora por el hecho de que existen pérdidas en la propagación del sonido menores a las esperadas en estos horarios. No hubo interferencia significativa del área del fragmento y de la hora en las respuestas de Callithrix spp. Sin embargo, algunos períodos del día tuvieron una mayor frecuencia de respuestas y, por lo tanto, la reproducción del playback puede ser útil para futuras encuestas de poblaciones de Callithrix, y se recomienda hacerlo preferencialmente en estos horarios en particular.
\end{abstract}

Palabras-clave: Callithrix aurita; fragmentación; vocalización; especie amenazada; especies invasoras.

\section{Introdução}

O sagui-da-serra-escuro ou saguicaveirinha (Callithrix aurita (E. Geoffroy, 1812)) é um pequeno primata endêmico do bioma Mata Atlântica dos estados de São Paulo, Minas Gerais e Rio de Janeiro (Melo et al., 2020). Atualmente, a espécie é classificada como 'Em Perigo' (EN), tanto pelo Livro Vermelho da Fauna Ameaçada de Extinção, quanto pela The IUCN Red List of Threatened Species $^{\mathrm{TM}}$ (Melo et al., 2018; Melo et al., 2020). Dentre as principais ameaças à sua conservação, destaca-se o desmatamento da Mata Atlântica (Valverde, 1958), resultando na redução, degradação e fragmentação de seu habitat (ICMBio, 2018; Melo et al., 2018; Melo et al., 2020). Tal ameaça vem causando o isolamento das populações remanescentes, deixando-as susceptíveis a eventos deletérios, como a endogamia (Santana et al., 2008; Vale, 2016). Outra grande ameaça é a introdução de espécies alóctones do gênero Callithrix dentro de sua área de distribuição, frequentemente em consequência do tráfico ilegal de animais silvestres (Mittermeier, 1982; Carvalho et al., 2018) e de solturas indevidas (Silva et al., 2018). Devido à proximidade filogenética, as espécies do gênero Callithrix podem acasalar entre si, formando descendentes híbridos férteis e, com isso, causando a erosão genética das populações de C. aurita (Carvalho et al., 2018; Silva et al., 2018; Melo et al. 2020).

As estratégias para a conservação de $C$. aurita foram inicialmente estabelecidas no Plano de Ação Nacional para a Conservação dos Mamíferos da Mata Atlântica Central (PAN MAMAC), em 2010, e posteriormente atualizadas no PAN para a Conservação dos Primatas da Mata Atlântica e da Preguiça-de-Coleira (PAN PPMA), em 2018. Entre tais estratégias, consta o mapeamento e a identificação de fragmentos florestais habitados por populações remanescentes da espécie, seus congêneres invasores $(C$. jacchus, $C$. penicillata, C. geoffroyi), além de populações híbridas (Mendes et al., 2016; Melo et al., 2018; ICMBio, 2019). Essas informações são fundamentais para obter um diagnóstico atualizado sobre a situação da espécie, bem como para subsidiar a tomada de decisões de manejo de habitat e populações e o desenvolvimento de programas para mitigação das ameaças e melhoria do estado de conservação da espécie (Carvalho et al., 2019).

As populações remanescentes de Callithrix aurita podem estar subamostradas pelas técnicas tradicionais de censo normalmente usadas em levantamentos populacionais (Muskin, 1984). Isso pode ocorrer devido ao pequeno tamanho 
corporal dos indivíduos de C. aurita, sua coloração críptica e movimentos rápidos, o que dificulta seu reconhecimento em campo, além de sua preferência por áreas densas de bambu e emaranhados de cipós (Muskin, 1984). Na cidade de Viçosa/MG, por exemplo, a espécie foi considerada extinta localmente por Pereira (2012), que realizou um levantamento de espécies de primatas utilizando apenas o método de transecção linear. Entretanto, alguns poucos indivíduos da espécie foram avistados por Vital (2017) em fragmentos florestais da região através de técnica de busca ativa com auxílio de playback - a reprodução de vocalizações para estimular a resposta de animais residentes (Dacier et al., 2011). Desta forma, a eficiência de diferentes métodos de levantamento populacional deve ser levada em consideração para a definição das técnicas a serem utilizadas em campo (Brasileiro, 2019), principalmente quando a amostragem de presença/ausência, abundância e/ou densidade tem como foco espécies crípticas e elusivas (Paranhos, 2006).

O presente estudo teve como objetivo avaliar a eficácia do playback para levantamentos populacionais de C. aurita, congêneres invasores $e$ híbridos com relação às seguintes hipóteses: a) a taxa de resposta de indivíduos de Callithrix spp. à emissão de playback depende do tamanho do fragmento; e b) a taxa de resposta de indivíduos de Callithrix spp. à emissão de playback depende do horário.

\section{Material e Métodos}

A microrregião de Viçosa está localizada na Zona da Mata do estado de Minas Gerais (Figura 1), que recebe essa denominação por sua cobertura vegetal original ser predominantemente de Mata Atlântica. A microrregião é composta por 20 municípios que totalizam $4.825 \mathrm{~km}^{2}$, com uma população de 221.630 habitantes (IBGE, 2019). O clima médio da região, de acordo com a classificação de Köppen, varia entre Temperado Úmido com Inverno Seco e Verão quente (Cwa) e Subtropical Moderado Úmido (Cwb), com verões quentes e chuvosos e invernos frios e secos (Valverde, 1958; Antunes, 1986; Alvares et al., 2013).

Os fragmentos florestais para amostragem (Figura 1) foram selecionados a partir de imagens de satélite disponíveis no programa Google Earth Pro e na plataforma Google Maps, utilizando-se para marcação dos pontos de busca o aplicativo (c)Wikiloc. Os fragmentos foram selecionados de forma aleatória dentro dos limites dos municípios pertencentes à microrregião de Viçosa, sendo excluídos fragmentos vistoriados em outros levantamentos similares recentes, como Pereira (2012) e Vital (2017).

As campanhas foram realizadas entre agosto de 2018 e julho de 2019 em dois períodos: matutino, a partir das $6 \mathrm{~h}$ até às $12 \mathrm{~h}$, e vespertino, a partir das $14 \mathrm{~h}$ até às $18 \mathrm{~h}$ (Melo, 2008). Para coleta dos dados sobre presença ou ausência tanto de C. aurita quanto de outras espécies de saguis, foi utilizado o método de busca ativa com auxílio de playback. Esse método consistiu em caminhadas a uma velocidade média de $1,5 \mathrm{~km} / \mathrm{h}$ com a emissão periódica de playback, tanto nas bordas dos fragmentos florestais, quanto em seu interior, utilizando-se, para tal, trilhas e acessos pré-existentes (Garcia et al., 2014).

Nas sessões de playback, foram reproduzidas vocalizações longas (long-calls, também conhecidas como phee calls) de Callithrix aurita (obtidas in situ por FRM). Essas vocalizações são usadas pelos calitriquídeos para diversas funções sociais, como localização de indivíduos, defesa de território e reprodução (Snowdon, 1993). Para tal, foi usado uma caixa amplificadora de som (Bluetooth modelo Q3 com potência de 5W, ou JBL Clip 2 com potência de $3 \mathrm{~W}$ ) e um aparelho reprodutor de arquivos de áudio (Kierulff \& Rylands, 2003; Jerusalinsky, 2013). Os playbacks foram realizados com distância entre os pontos de, aproximadamente, $100 \mathrm{~m}$, e conduzidos em sessões alternadas, sendo 3 sessões de 2 minutos cada de playback ativo, com intervalos entre as sessões de 2 minutos cada, totalizando 12 minutos em cada ponto.

Quando houve respostas ao playback no ponto amostrado ou foram registradas vocalizações espontâneas durante o percurso entre os pontos, realizou-se busca ativa visando a observação direta dos animais e a identificação da espécie do gênero Callithrix que estava presente na área. Para cada indivíduo ou grupo registrado, foram anotadas informações como tamanho e composição sexoetária dos grupos e sua posição georreferenciada por meio de equipamento receptor de GPS. Os resultados foram consolidados em mapas com o aplicativo Wikiloc e Google Earth Pro com as localizações dos registros em relatório diário. 
Para a diferenciação das espécies de Callithrix, utilizou-se o método de observação direta e registros fotográficos dos animais em campo, com o uso de câmera digital profissional (Canon EOS Rebel T6, lentes 55-250mm, e Nikon D5200, lentes 70-300mm). A partir dessas imagens e das observações diretas, foram analisadas as características morfológicas, especialmente de coloração da pelagem, utilizando os padrões descritos para cada espécie e os caracteres diagnósticos descritos por Mendes (1997) e Moraes \& Melo (2011).

Com as informações coletadas in situ criou-se um banco de dados sobre as áreas de ocorrência de Callithrix spp. - nativos, invasores e híbridos -, o contexto local dos fragmentos vistoriados, $e$ as principais ameaças às populações remanescentes de Callithrix aurita. Com esse conjunto de informações foi possível avaliar a atual situação de $C$. aurita na região de estudo e propor recomendações de manejo para sua conservação. Aplicou-se um teste chi-quadrado com nível de significância $5 \%$ e dois graus de liberdade para averiguar a relação entre a área dos fragmentos amostrados (classificados em: menores de 50ha; maiores que 50 e menores que 100ha; maiores que 100ha).

\section{Resultados}

Ao todo, 15 fragmentos foram vistoriados em sete municípios da microrregião de Viçosa/ MG, com áreas de sete (07) a 180ha (Figura 1). As vistorias demandaram 13 campanhas e 61 horas de campo (em média 4:06 horas para cada fragmento) para a marcação de 88 pontos de playback (em média, seis pontos marcados por fragmento) (Tabela 1). Obteve-se resposta para apenas cinco $(5,68 \%)$ das execuções de playback, sendo todas estas entre $07 \mathrm{~h}$ e $08 \mathrm{~h}$ da manhã (60\%) ou entre 16 e $17 \mathrm{~h}$ da tarde (40\%) (Tabela 2, Figura 2). Não houve detecção de vocalizações espontâneas, ou seja, todas as vocalizações registradas foram em resposta à execução do playback.

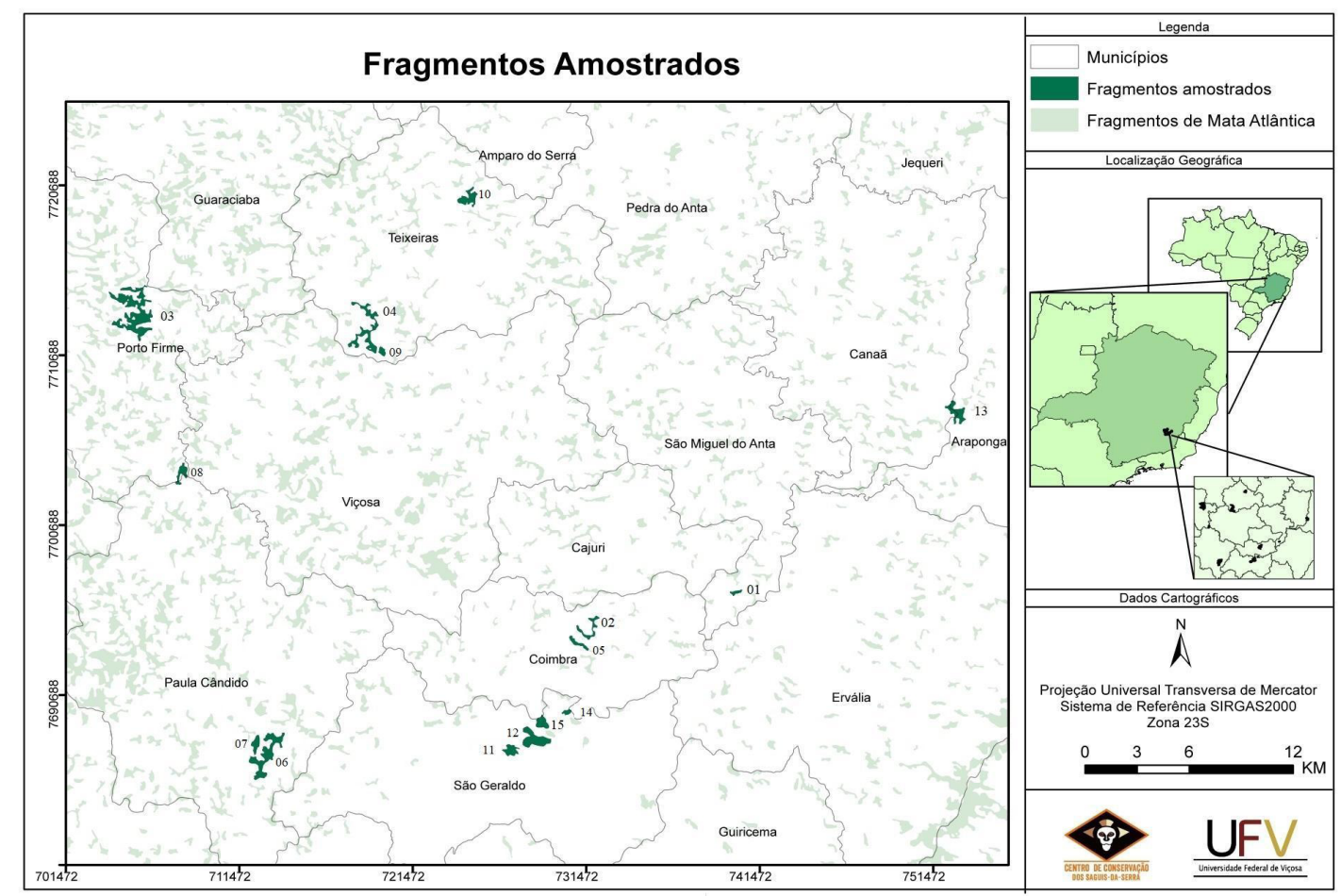

Figura 1 - Localização dos fragmentos florestais amostrados na microrregião de Viçosa. 
Tabela 1 - Identificação dos fragmentos com as respectivas cidades, pontos de playback realizados e a área, em hectares.

\begin{tabular}{|c|c|c|c|c|}
\hline Id. & Município & Coordenadas geográficas & Pontos de playback & Área (ha) \\
\hline 1 & São Miguel do Anta & $20^{\circ} 48^{\prime} 51.89^{\prime \prime S}, 42^{\circ} 41^{\prime} 34.29^{\prime \prime O}$ & 7 & 15 \\
\hline 2 & Coimbra & 2050’17.87”S, 4246’35.17”O & 7 & 60 \\
\hline 3 & Porto Firme & $20^{\circ} 40^{\prime} 34.49^{\prime \prime} \mathrm{S}, 43^{\circ} 1^{\prime} 39.85^{\prime \prime O}$ & 12 & 180 \\
\hline 4 & Teixeiras & $20^{\circ} 40^{\prime} 7.56^{\prime \prime S}, 42^{\circ} 53^{\prime} 53.48^{\prime \prime O}$ & 5 & 90 \\
\hline 5 & Coimbra & $20^{\circ} 50^{\prime} 28.18^{\prime \prime} \mathrm{S}, 42^{\circ} 46^{\prime} 49.13^{\prime \prime O}$ & 1 & 30 \\
\hline 6 & Paula Cândido & $20^{\circ} 54^{\prime} 3.69^{\prime \prime} \mathrm{S}, 42^{\circ} 57^{\prime} 3.05^{\prime \prime} \mathrm{O}$ & 7 & 175 \\
\hline 7 & Paula Cândido & $20^{\circ} 53^{\prime} 32.74 ” S, 42^{\circ} 57^{\prime} 39.92^{\prime \prime O}$ & 7 & 55 \\
\hline 8 & Porto Firme & $20^{\circ} 44^{\prime} 59.10^{\prime \prime} \mathrm{S}, 42^{\circ} 59^{\prime} 53.21^{\prime \prime} \mathrm{O}$ & 1 & 55 \\
\hline 9 & Teixeiras & $20^{\circ} 41^{\prime} 11.67 ” S, 42^{\circ} 53^{\prime} 31.93$ ”O & 6 & 145 \\
\hline 10 & Teixeiras & $20^{\circ} 36^{\prime} 26.23^{\prime \prime} \mathrm{S}, 42^{\circ} 50^{\prime} 37.63^{\prime \prime O}$ & 4 & 110 \\
\hline 11 & Coimbra/ São Geraldo & $20^{\circ} 53^{\prime} 34.90^{\prime \prime} \mathrm{S}, 42^{\circ} 48^{\prime} 24.35^{\prime \prime O}$ & 6 & 7 \\
\hline 12 & Coimbra/São Geraldo & $20^{\circ} 53^{\prime} 12.51^{\prime \prime S}, 42^{\circ} 48^{\prime} 20.43^{\prime \prime O}$ & 5 & 20 \\
\hline 13 & Canaã & $20^{\circ} 42^{\prime} 59.74^{\prime \prime} \mathrm{S}, 42^{\circ} 35^{\prime} 55.20^{\prime \prime} \mathrm{O}$ & 8 & 30 \\
\hline 14 & Coimbra & $20^{\circ} 52^{\prime} 44.54^{\prime \prime S}, 42^{\circ} 47^{\prime} 8.64^{\prime \prime} \mathrm{O}$ & 7 & 25 \\
\hline 15 & Coimbra/São Geraldo & $20^{\circ} 52^{\prime} 55.88^{\prime \prime} \mathrm{S}, 42^{\circ} 47^{\prime} 52.52^{\prime \prime O}$ & 5 & 65 \\
\hline
\end{tabular}

Tabela 2 - Número de respostas de Callithrix spp. aos playbacks executados, de acordo com a hora do dia.

\begin{tabular}{|l|c|c|c|}
\hline \multicolumn{2}{|c|}{ Manhã } & & Tarde \\
\hline Hora do dia & $\begin{array}{c}\text { Número de fragmentos com } \\
\text { respostas }\end{array}$ & Hora do dia & $\begin{array}{c}\text { Número de fragmentos com } \\
\text { respostas }\end{array}$ \\
\hline $07: 00-07: 59$ & 3 & $14: 00-14: 59$ & 0 \\
\hline $08: 00-08: 59$ & 0 & $15: 00-15: 59$ & 0 \\
\hline $09: 00-09: 59$ & 0 & $16: 00-16: 59$ & 2 \\
\hline $10: 00-10: 59$ & 0 & $17: 00-17: 59$ & 0 \\
\hline $11: 00-11: 59$ & 0 & & \\
\hline
\end{tabular}

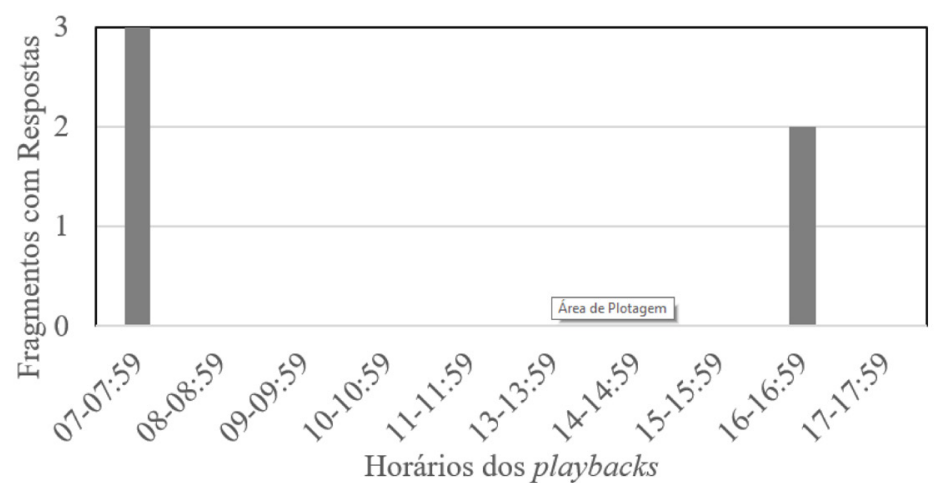

Figura 2 - Gráfico ilustrando o número de fragmentos com respostas de Callithrix spp. em relação aos horários de playbacks executados. 
Não houve registros de C. aurita em nenhum fragmento amostrado, por outro lado, foram obtidos 11 registros da ocorrência de Callithrix spp. em cinco $(33,33 \%)$ dos fragmentos amostrados, sendo sete por observação direta $e$ quatro por vocalizações. Nas observações diretas e com a análise dos registros fotográficos, foi possivel identificar três híbridos entre C. aurita e Callithrix spp. (Figura 3). Dentre os cinco fragmentos com ocorrência registrada de spp., três deles (60\%) possuem área maior que 100 ha (fragmentos 03 , 06, 10 - Figura 1), um (20\%) tem área entre 50 e 100ha (fragmento 08) e um (20\%) tem área menor que 50ha (fragmento 11) (Tabela 3).
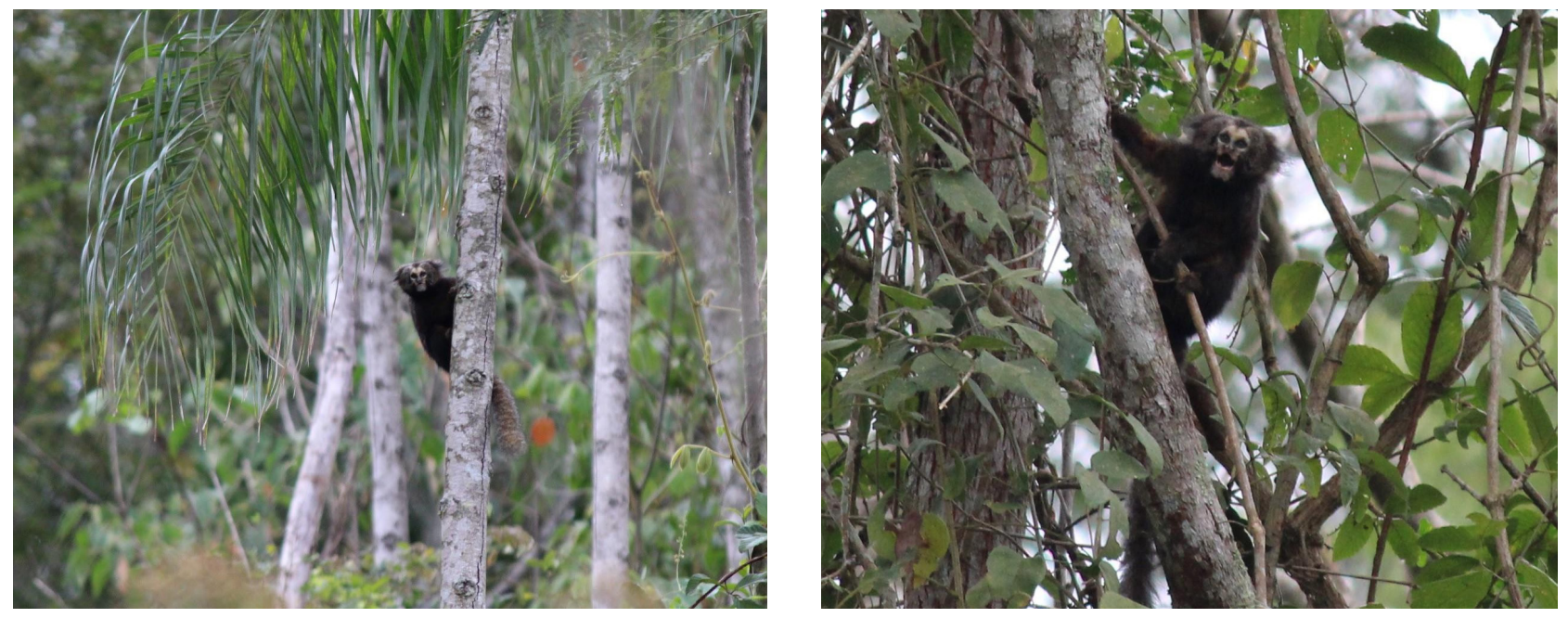

Figura 3 - Indivíduos híbridos entre Callithrix aurita e outra espécie do gênero. Fotos: Natan Massardi.

As maiores frequências de encontro de Callithrix spp. ocorreram em fragmentos acima de 100 ha $(75 \%$ dos amostrados acima de 100ha), seguido por fragmentos entre 50 e 100ha (20\%) e, por último, fragmentos menores que 50ha $(16,7 \%)$ (Tabela 3). Algumas áreas dos fragmentos selecionados não puderam ser amostradas por se tratar de propriedades privadas e o respectivo proprietário não estar presente para autorizar a entrada.

Tabela 3 - Frequência de encontros de Callithrix spp. em relação à área do fragmento.

\begin{tabular}{|l|l|l|l|}
$\begin{array}{c}\text { Área do } \\
\text { fragmento } \\
\text { (ha) }\end{array}$ & $\begin{array}{c}\text { Número de } \\
\text { fragmentos } \\
\text { vistoriados }\end{array}$ & $\begin{array}{c}\text { Fragmentos com } \\
\text { observação } \\
\text { direta de } \\
\text { Callithrix sp. }\end{array}$ & $\begin{array}{c}\text { Frequência } \\
\text { de presença } \\
\text { por área }\end{array}$ \\
\hline$>100$ & 4 & 3 & $75 \%$ \\
\hline$>50,<100$ & 5 & 1 & $20 \%$ \\
\hline$<50$ & 6 & 1 & $16,7 \%$ \\
\hline
\end{tabular}

\section{Discussão}

A técnica de playback é uma ferramenta útil para o levantamento de algumas espécies de primatas, como as espécies de Callithrix e Callicebus, uma vez que essas tendem a responder à reprodução das vocalizações, o que possibilita sua detecção, ampliando as chances de observação direta por parte do pesquisador (Mendes, 1997; Melo \& Mendes, 2000; Bezerra et al., 2017).

Os fragmentos amostrados apresentaram características de mata secundária, de maneira que suas bordas eram dominadas por vegetação densa, o que dificultou ou impediu o acesso ao interior dos mesmos. A grande densidade do sub-bosque presente nesses fragmentos pode ter influenciado também na propagação do som do playback, uma vez que os saguis apresentam vocalizações de alta frequência que se dissipam com menor eficiência ao longo das matas (Soares et al., 2011). Sendo assim, normalmente apenas grupos que estão mais próximos do ponto de emissão do som do playback tendem a respondêlo (Soares et al., 2011). 
Pode-se inferir que a ausência de respostas por grupos de saguis nos fragmentos amostrados pode ser consequência do histórico de colonização humana e desmatamento das grandes áreas de Mata Atlântica da região nos séculos XIX e XX (Valverde, 1958; Dean, 1996). A partir desse cenário, presume-se que Callithrix aurita, espécie nativa da região, sofreu grande redução populacional, mantendo-se em fragmentos remanescentes e/ou povoando fragmentos de mata secundária (Silva, 2018; Vital, 2020). Os inúmeros fragmentos de mata secundária presentes na região podem ainda não ter sido colonizados em sua totalidade tanto pela espécie nativa, cuja população fora reduzida drasticamente, quanto pelas populações de saguis invasores e formas híbridas, justificando a baixa taxa de resposta ao playback encontrada no presente estudo.

A prevalência de respostas ao playback nos horários iniciais da manhã (das 07 às $08 \mathrm{~h}$ ) e ao entardecer (das 16 às $17 \mathrm{~h}$ ), como registrados neste estudo, é corroborada pelo fato do som se propagar melhor nestes horários em ambientes florestais, diminuindo a perda de frequências $e$ aumentando as chances do playback ser ouvido $e$ respondido. Essa perda é influenciada pelas propriedades dinâmicas da formação ou dissipação de gradientes de temperatura nesses períodos. Desse modo, as copas das árvores formam uma barreira física e um tampão microclimático favorecendo a propagação do som (Ingard, 1953; Mendes, 1997). Nos períodos mais quentes do dia, a temperatura favorece a atenuação do som (Ingard, 1953), o que pode explicar a preferência de vocalizações no início da manhã e no final da tarde (Silva, 2013).

Apesar de as áreas de vida de Callithrix spp. apresentarem grandes variações de tamanho nas diferentes espécies - desde 0,5ha, para $C$. jacchus, até 130 ha em C. flaviceps (Passamani \& Rylands, 2000; Hilário, 2009) -, em C. aurita variam de 11 a 60,84ha (Muskin, 1984; Palacios, 2018). Dentre os resultados, obtivemos maior taxa de encontros de Callithrix spp. em fragmentos maiores que 100 ha com relação às demais classes de tamanho (entre 50ha e 100ha e menores que 50ha). Isso pode estar relacionado ao histórico de uso e ocupação do território da microrregião de Viçosa, já que em 1998 cerca de $74 \%$ da área regional total era ocupada por pastagens $e$ agropecuária, e os fragmentos remanescentes restaram isolados em áreas de difícil acesso $e$ relevo acidentado (Valverde, 1958; Faria et al., 2009). Esses fragmentos remanescentes de maior tamanho podem ter servido como refúgios para os animais (principalmente os arborícolas), mas, por outro lado, resultaram no isolamento populacional nessas "ilhas" (Coelho et al., 2005). Todavia, após a realização do teste chi-quadrado $\left(\mathrm{X}_{(15 ; 2)}^{2}, \mathrm{p}=\right.$ $0,398)$, não foi possível correlacionar a área dos fragmentos vistoriados com a taxa de resposta de Callithrix spp. ao playback.

$\mathrm{O}$ fato de a presente pesquisa não ter sido executada em fragmentos anteriormente amostrados por outros estudos, e que indicam a presença de grupos puros e mistos de C. aurita, dificulta uma análise sobre os efeitos da fragmentação nas populações de sagui. Porém, acredita-se que a ausência de grupos puros de C. aurita na área de estudo esteja relacionada com a grande redução, degradação e fragmentação da Mata Atlântica e sua substituição por pastagens $e$ agropecuária nesta região. A presença de poucos grupos de $C$. aurita na microrregião de Viçosa alerta ainda para a importância da proteção e restauração dos remanescentes florestais visando ampliar as condições para a manutenção de populações viáveis dessa espécie (Silva et al., 2018; Vital, 2020). Para tal, futuros levantamentos das populações de Callithrix, com uso de playback orientado pelos resultados obtidos, podem ajudar a direcionar os esforços para a conservação de $C$. aurita.

\section{Conclusão}

Houve baixa taxa de resposta de Callithrix spp. ao playback, em apenas cinco $(5,85 \%)$ de 88 sessões e cinco (33,3\%) de 15 fragmentos. Não houve influência da área total dos fragmentos florestais amostrados nas taxas de resposta de Callithrix spp. ao playback. Já com relação ao horário das respostas com vocalizações ao playback, apesar de não ter sido identificada diferença significativa entre as taxas de resposta pela manhã e à tarde, foram identificados dois horários com maior frequência de respostas: das $07 \mathrm{~h}$ às $08 \mathrm{~h}$; das $16 \mathrm{~h}$ às $17 \mathrm{~h}$.

Assim, a técnica de playback pode ser útil para futuros levantamentos das populações de Callithrix, em fragmentos já amostrados e não amostrados, independentemente de seu tamanho. Entretanto, recomenda-se o direcionamento das sessões de playback para os horários com maiores 
taxas de resposta, o que pode gerar informações valiosas para implementar os esforços de manejo para controle de invasores e híbridos e para a conservação de $C$. aurita.

\section{Agradecimentos}

Ao Programa Institucional de Bolsas de Iniciação Científica (PIBIC) do ICMBio e ao CNPq, pela concessão da bolsa de estudos de iniciação científica, crucial para a realização das campanhas de campo e manutenção de equipamentos. À UFV e à equipe do CCSS, pelo apoio logístico para execução das atividades.

\section{Referências}

Alvares, CA; Stape, JL; Sentelhas, PC; Gonçalves, JLM; Sparovek, G. Köppen's climate classification map for Brazil. Meteorologische Zeitschrift, v. 22, n. 6, p. 711-728, 2013.

Antunes FZ. Caracterização climática do estado de Minas Gerais. Informe Agropecuário, 138: 84, 1986.

Bezerra B. et al. Pitheciid vocal communication: What can we say about what they are saying? Ethnobiology and Conservation, v. 6, n. 15. September, p. 1-23, 2017.

Brasileiro SLS. 2019. Avaliação da eficiência do método de playback para primatas em comparação ao método tradicional de transecto linear: uma revisão sistemática. Monografia. Universidade Federal de Viçosa. 34p.

Carvalho RS et al. Callithrix aurita: a marmoset species on its way to extinction in the Brazilian Atlantic Forest. Neotropical Primates, 24(1): 1-8, 2018.

Carvalho, RS. et al. Buffy-Tufted-Ear-Marmoset Callithrix aurita É. Geoffroyi Saint-Hillaire, 1812. In: Schwitzer, C. et al. Primates in Peril: The World's 25 Most Endangered Primates 2018-2020. IUCN SSC Primate Specialist Group, International Primatological Society, Global Wildlife Conservation, and Bristol Zoological Society, Washington, DC, p. 79-81, 2019.

Coelho DJ Da S; Souza AL De S, Oliveira CML De. Levantamento da Cobertura Florestal Natural da Microrregião de Viçosa, MG, Utilizando-se Imagens de LANDSAT 5. R. Árvore, Viçosa-MG, v.29, n.1, p.17-24, 2005.

Dacier A, Luna AG, Fernandez-Duque E, Di Fiore A. Estimating Population Density of Amazonian Titi Monkeys (Callicebus discolor) via Playback Point Counts. Biotropica 43(2): 135-140, 2011.
DEAN, W. A ferro e fogo: A história e a devastação da Mata Atlântica brasileira. São Paulo: Companhia das Letras, 484p, 1996.

Faria $\mathrm{AL}$ et al. Um novo olhar sobre a cidade: a experiência de construção do atlas escolar histórico e geográfico de viçosa (mg) 5. Revista de C. Humanas, 9(1): 67-84, 2009.

Garcia VLA, Fialho MS, Jerusalinsky L. Uso de playback para levantamento populacional de Alouatta belzebul (Linnaeus, 1766) reintroduzidos na Reserva Biológica Guaribas, Paraíba. A Primatologia no Brasil, 2014.

Hilário RR. 2009. Padrão de atividades, dieta e uso do hábitat por Callithrix flaviceps na Reserva Biológica Augusto Ruschi, Santa Teresa, ES. Dissertação (Mestrado em Ecologia). Universidade Federal de Minas Gerais. 124p.

IBGE. Diretoria de Pesquisas - DPE - Coordenação de População e Indicadores Sociais - COPIS. 2019. Disponível em: <ftp://ttp.ibge.gov.br/Estimativas_de_Populacao/ Estimativas_2019/estimativa_TCU_2019_20200116. pdf $>$. Acesso em: 13/08/2020.

Ingard U. A review of the influence of meteorological conditions on sound propagation. The Journal of the Acoustical Society of America. 25(3): 405-411, 1953.

Instituto Chico Mendes de Conservação da Biodiversidade/ICMBio. Sumário Executivo do Plano de Ação Nacional para a Conservação dos Primatas da Mata Atlântica e da Preguiça-de-Coleira. 9p. 2019. Disponível em <https://www.icmbio.gov.br/portal/ images/stories/docs-pan/pan-primatas-e-preguica-da$\mathrm{ma} / 1$-ciclo/pan-primatas-e-preguica-da-ma-sumario. pdf $>$ Acesso em 14/08/2020.

Jerusalinsky L. 2013. Distribuição geográfica e conservação de Callicebus coimbrai (Primates Pitheciidae) na Mata Atlântica do nordeste do Brasil. Tese (Doutorado em Zoologia). Universidade Federal da Paraíba. 272p.

Kierulff MCM, Rylands AB. Census and distribution of the golden lion tamarin (Leontopithecus rosalia). American Journal of Primatology, 59(1): 29-44, 2003.

Melo FR. et al. 2018. Callithrix aurita (É. Geoffroy Saint-Hilaire, 1812). In: Instituto Chico Mendes de Conservação da Biodiversidade. (org.). Livro Vermelho da Fauna Brasileira Ameaçada de Extinção: Volume II Mamíferos. Brasilia: ICMBio. p. 206-213.

Melo FR. et al. 2020. Callithrix aurita (amended version of 2019 assessment). The IUCN Red List of Threatened Species 2020: e.T3570A166617776. https://dx.doi.org/10.2305/IUCN.UK.2020-1.RLTS. T3570A166617776.en. Downloaded on 14 August 2020. 
MELO FR; MENDES, SL. Emissão de gritos longos por grupos de Callicebus nigrifrons e suas reações a playbacks, p.215-222, 2000. In: C. ALONSO \& A. LANGGUTH (Eds). A Primatologia do Brasil 7. João Pessoa, Sociedade Brasileira de Primatologia e Editora Universitária.

Melo FR; Rylands AB. Callithrix aurita - Livro Vermelho da Fauna Brasileira Ameaçada de Extinção. Livro Vermelho da Fauna Brasileira Ameaçada de Extinção, n. 1983, p. 735-737, 2008.

Mendes SL. 1997. Padrões Biogeográficos e Vocais em Callithrix do Grupo jacchus (Primates, Callitrichidae). Tese (Doutorado em Ecologia), Universidade Estadual de Campinas, Campinas, SP. 155p.

Mendes SL, Brandão LD, Igayara C. Callithrix aurita (E. Geoffroy in Humboldt, 1812). Plano de Ação Nacional para Conservação dos Mamíferos da Mata Atlântica Central, 142-147, 2016.

Mittermeier RA, Coimbra - Filho AF, Constable ID, Rylands, $\mathrm{AB}$, Valle $\mathrm{C}$. Conservation of primates in the Atlantic forest region of eastern Brazil. International Zoo Yearbook, 22(1): 2-17, 1982.

Moraes AM, Melo FR. Distribuição geográfica de Callithrix aurita e Callithrix flaviceps e avaliação espacial de sua zona de intergradação nos municípios de Espera Feliz, Caiana e Caparaó, MG. A Primatologia no Brasil. p. 231-255, 2011.

Muskin A. Field notes and geographical distribution of Callithrix aurita in eastern Brazil. American Journal of Primatology, 7(4):377-380, 1984.

Palacios AMS. 2018. Efeito de fatores ambientais e ecológicos nas áreas de vida do "sagui-da-serra-escuro" (Callithrix aurita) na Mata Atlântica. Dissertação (Mestrado em Ecologia). Universidade Estadual de Campinas. 64p.

Paranhos KM. 2006. Estimativas populacionais para espécies raras: o mico-leão-preto Leontopithecus chrysopygus (Mikan, 1823) como modelo. Dissertação (Mestrado em Ecologia e Conservação). Universidade Federal do Paraná. 53p.

Passamani M, Rylands AB. Home Range of a Geoffroy's Marmoset Group, Callithrix geoffroyi (Primates, Callitrichidae) in South-Eastern Brazil. Revista Brasileira de Biologia, v. 60, p. 275-281, 2000.

Pereira AM. 2012.Composição, Distribuição, Densidade e Riqueza De Primatas Em Fragmentos Florestais No Município De Viçosa-Mg. Universidade Federal de Viçosa. Dissertação (Mestrado em Biologia Animal). 81p.
Rylands AB, Fonseca GAB, Leite YLR, Mittermeier RA. 1996. Primates of the Atlantic Forest: origin, distributions, endemism, and communities, p.21-51. In: Adaptive Radiations of Neotropical Primates, M. A. Norconk, A. L. Rosenberger and P. A. Garber (eds.), Plenum Press, New York.

Santana BEMM et al. Densidade, tamanho populacional e abundância dos primatas em um fragmento de floresta atlântica em Minas Gerais, Brasil. Revista Árvore, 32 (6):1009-1117, 2008.

Silva FFR et al. A Survey of Wild and Introduced Marmosets (Callithrix: Callitrichidae) in the Southern and Eastern Portions of the State of Minas Gerais, Brazil. Primate Conservation, 32:1-18, 2018.

Silva OC. 2013. Um estudo comparativo sobre a propagação do phee-call do sagui-comum em Caatinga e Mata Atlântica no Nordeste do Brasil. Dissertação (Mestrado em Ecologia). Universidade Federal Rural de Pernambuco. 52p.

Snowdon C. A vocal taxonomy of the callitrichids. Marmosets and Tamarins: Systematics, behaviour, and ecology, n. July, p. 78-94, 1993.

Soares NM, Santos Jr, EM, Beltrão-Mendes R, Ferrari SF. Avaliação preliminar de uso de habitat e reações ao playback em Callicebus coimbrai Kobayashi \& Langguth, 1999 e Callithrix jacchus (Linnaeus, 1758) no Refúgio de Vida Silvestre Mata do Junco, Sergipe, 2011.

Vale CA. Distribuição e potencial de invasão do sagui Callithrix penicillata (É. Geoffroy, 1812) no território brasileiro. 2016. Universidade Federal de Juiz de Fora.

Valverde O. Estudo Regional da Zona da Mata de Minas Gerais. Revista Brasileira de Geografia, p. 3-82, 1958.

Vital OV. 2017.Ocorrência do Sagui-da-SerraEscuro Callithrix aurita Humboldt, 1812 (Primates, Callitrichidae), na microrregião de Viçosa, Zona da Mata - MG. Monografia, Universidade Federal de Viçosa, Viçosa, MG. 38p.

Vital OV. 2020. Uso e ocupação do hábitat por Callithrix spp. em remanescentes de mata atlântica na microrregião de Viçosa, Minas Gerais. Dissertação (Mestrado em Biologia Animal), Universidade Federal de Viçosa, Viçosa, MG. 45p.

(C) Wikiloc. Trilhas do Mundo. Disponível em: https:// pt.wikiloc.com. Acesso em: 13 abr. 2021. 


\section{Biodiversidade Brasileira - BioBrasil. \\ Edição Temática: PIBIC}

n. 1,2022

http://www.icmbio.gov.br/revistaeletronica/index.php/BioBR

Biodiversidade Brasileira é uma publicação eletrônica científica do Instituto Chico Mendes de

Conservação da Biodiversidade (ICMBio) que tem como objetivo fomentar a discussão e a disseminação de experiências em conservação e manejo, com foco em unidades de conservação $e$ espécies ameaçadas.

ISSN: 2236-2886 